\title{
A MARKOVIAN GROWTH-COLLAPSE MODEL
}

\author{
ONNO BOXMA, * EURANDOM and Eindhoven University of Technology \\ DAVID PERRY, ${ }^{* *}$ University of Haifa \\ WOLFGANG STADJE, ${ }^{* * *}$ University of Osnabrück \\ SHELEMYAHU ZACKS, ${ }^{* * * *}$ Binghamton University
}

\begin{abstract}
We consider growth-collapse processes (GCPs) that grow linearly between random partial collapse times, at which they jump down according to some distribution depending on their current level. The jump occurrences are governed by a state-dependent rate function $r(x)$. We deal with the stationary distribution of such a GCP, $\left(X_{t}\right)_{t \geq 0}$, and the distributions of the hitting times $T_{a}=\inf \left\{t \geq 0: X_{t}=a\right\}, a>0$. After presenting the general theory of these GCPs, several important special cases are studied. We also take a brief look at the Markov-modulated case. In particular, we present a method of computing the distribution of $\min \left[T_{a}, \sigma\right]$ in this case (where $\sigma$ is the time of the first jump), and apply it to determine the long-run average cost of running a certain Markov-modulated disaster-ridden system.
\end{abstract}

Keywords: Growth-collapse process; piecewise-deterministic Markov process; stationary distribution; hitting time; uniform cut-off; duality; Markov modulation

2000 Mathematics Subject Classification: Primary 60K30

Secondary 60J27; 60J75; 60F05

\section{Introduction}

Growth-collapse processes (GCPs) are real-valued stochastic processes that grow (possibly in a random fashion) between random collapse times, at which they jump down according to some distribution depending on their current level. This pattern of behavior can be encountered in a large variety of physical phenomena, for example the build-up of friction, earthquakes, avalanches, and neuron firing; cf. Bak's [7, pp. 33-48] paradigm of 'systems of self-organized criticality'. Also, in population growth models it seems reasonable to assume that the growth rate and the extent of occasional 'disasters' (e.g. epidemics) depend on the current population size. In the realm of operations research, GCPs occur in insurance mathematics and related fields, and in models of production/inventory systems (see [5, Chapter VI], [6, pp. 399-406], or [22, Chapters 5 and 11]) and queueing (see, e.g. [8]).

Eliazar and Klafter [16] considered a GCP, $X=\left(X_{t}\right)_{t \geq 0}$, composed of three random sources: (a) a steady, random inflow with stationary, independent, positive increments; (b) crash times $\tau_{1}<\tau_{2}<\ldots$ that form a renewal process; and (c) crash proportions $U_{1}, U_{2}, \ldots$ that are independent, identically distributed (i.i.d.) random variables on $(0,1)$. The three sources are

\footnotetext{
Received 2 September 2005; revision received 13 December 2005.

* Postal address: Department of Mathematics and Computer Science, Eindhoven University of Technology, PO Box 513, 5600 MB Eindhoven, The Netherlands. Email address: boxma@win.tue.nl

** Postal address: Department of Statistics, University of Haifa, Haifa, 31909, Israel. Email address: dperry@ haifa.ac.il

*** Postal address: Department of Mathematics and Computer Science, University of Osnabrück, 49069 Osnabrück, Germany. Email address: wolfgang@mathematik.uni-osnabrueck.de

**** Postal address: Department of Mathematical Sciences, Binghamton University, Binghamton, NY 13902-6000, USA. Email address: shelly@math.binghamton.edu
} 
assumed to be stochastically independent. At the $n$th crash time, $\tau_{n}$, the process jumps down by the amount $U_{n} X_{\tau_{n}-}$; thus, the new system level at $\tau_{n}$ is $X_{\tau_{n}}=\left(1-U_{n}\right) X_{\tau_{n}-}$. Eliazar and Klafter [16] computed several system characteristics (means, variances, Laplace transforms, and probability tails) and focused on crash proportions governed by power law distributions.

In the spirit of [16], in this paper we study a class of $[0, \infty)$-valued, piecewise-deterministic Markov process, say $X=\left(X_{t}\right)_{t \geq 0}$, characterized by the following features.

(i) $X$ increases linearly at rate 1 between jumps and is right continuous.

(ii) Given that $X_{t}=x$, the probability of a jump in $(t, t+\eta)$ is equal to $r(x) \eta+o(\eta)$, as $\eta \rightarrow 0$, where $r:(0, \infty) \rightarrow[0, \infty)$ is a continuous function.

(iii) If a jump occurs at time $t$ and $X_{t-}=x$, then the distribution of $X_{t}$ is $\mu_{x}$, which is a probability measure on $[0, x)$.

Since $X$ is assumed to be Markovian, it follows from (ii) that the conditional probability of having a jump in $(t, t+\eta)$, given $\left(X_{s}\right)_{0 \leq s \leq t}$, is equal to $r\left(X_{t}\right) \eta+o(\eta)$, while (iii) implies that the conditional distribution of $X_{t}$, given that a jump occurs at $t$ and given $\left(X_{s}\right)_{0 \leq s<t}$, is $\mu_{X_{t-}}$. If $X_{0}=0$ then the function $r(x)$ in (ii) is just the failure rate of the first jump time $\sigma=\inf \left\{t \geq 0: X_{t} \neq X_{t-}\right\}$, meaning that

$$
\mathrm{P}(\sigma>t)=\exp \left\{-\int_{0}^{t} r(x) \mathrm{d} x\right\}, \quad t \geq 0 .
$$

It is reasonable to assume that $\mathrm{P}(\sigma<\infty)=1$, which is equivalent to $\int_{0}^{\infty} r(x) \mathrm{d} x=\infty$. In most examples the probability measure $\mu_{x}$ has a density $p(x, y)$ on $(0, x)$ and possibly an atom at 0 . A particularly interesting case that we will study is that of 'uniform' crash proportions, i.e. in which $p(x, y)=x^{-1} 1_{(0, x)}(y)$, where $1_{A}$ denotes the indicator function of the set $A$. Another example is the age process, $\left(A_{t}\right)_{t \geq 0}$, of a standard renewal process that can be described as follows: it starts at $A_{0}=0$, increases at rate 1 between jumps, and returns to 0 at every jump; and a jump occurs in $(t, t+\eta)$ with probability $r\left(A_{t}\right) \eta+o(\eta)$, as $\eta \rightarrow 0$, where $r(\cdot)$ is the failure rate of the underlying distribution function.

As an alternative to the growth condition (i), we also consider the case in which $X$ increases linearly between jumps at a rate $c\left(J_{t}\right)$, where $\left(J_{t}\right)_{t \geq 0}$ is a modulating irreducible Markov chain having state space $\{1, \ldots, n\}$, and the rates $c(j)$ satisfy $c(1)>c(2)>\cdots>c(n) \geq 0$.

In a variety of examples we will in particular deal with the stationary distribution of $X$ and the distribution of the hitting time $T_{a}=\inf \left\{t \geq 0: X_{t}=a\right\}$. In Section 2 we present the general theory of these distributions. In the subsequent sections these results are applied to important special cases. In Section 3 we give explicit formulae in the case in which the jump rate is proportional to the level achieved, i.e. $r(x)=\lambda x$, and the jump sizes are uniformly distributed $\left(\mu_{x}([0, y))=\min [y / x, 1]\right)$. Moreover, we prove that $a^{-2} \log T_{a} \rightarrow \lambda / 2$ in probability as $a \rightarrow \infty$. In Section 4 we consider uniform cut-offs with Poisson jump arrivals. It is shown that the stationary distribution of $X$ is $\operatorname{Erlang}(2, \lambda)$ and that the Laplace transform (LT) of $T_{a}$ can be given in terms of degenerate hypergeometric functions. Furthermore, we derive explicit formulae for the first two moments of the GCP at fixed time $t$ and just after the $n$th jump. In Section 5 we consider GCPs for which the time periods between the jumps are i.i.d. random variables with an arbitrary distribution. Using a duality argument, we prove that the level of the GCP after its $n$th jump has the same distribution as the level of a certain shot noise process that has been studied before, in [8]. Using this connection, the LT of the stationary density of the GCP can be derived. Finally, in Section 6 we take a brief look at the Markov-modulated 
case, as described above. In particular, we present a method of computing the distribution of $\min \left[T_{a}, \sigma\right]$ in this case, and apply it to determine the long-run average cost of running a certain Markov-modulated disaster-ridden system.

The assumption of linear growth at rate 1 between the jumps can be extended to cover more general rate functions $g(x)$. This leads to a model in which, for any $x_{0} \geq 0$ and $t_{1}$ and $t_{0}, t_{1} \geq t_{0} \geq 0$, and conditional on $X_{t_{0}}=x_{0}$ and on $X$ not having jumps in $\left[t_{0}, t_{1}\right)$, during $\left[t_{0}, t_{1}\right)$ the process evolves according to the differential equation $\mathrm{d} X_{t} / \mathrm{d} t=g\left(X_{t}\right), X_{t_{0}}=x_{0}$. However, it becomes much more difficult to obtain closed-form solutions.

We close this section with a brief discussion of some related GCP models. The sample paths of GCPs of the above type look like those of risk processes. However, in classical risk theory the surplus process of an insurance portfolio increases linearly between claims (leading to downward jumps), and the claim sizes and claim intervals are independent. The authors are not aware of risk studies in which the claim size depends on the size of the portfolio at the claim time. However, several authors did recently look at more general models in which the independence assumption is relaxed. See [5, Chapter VI] for a survey of the subject. In [1] the distribution of the claim interval depends on the previous claim size (see [9] for a queueing model with a similar dependence structure). In [2] a more general, semi-Markovian dependence structure is introduced in the risk model. The claim sizes now depend on some underlying Markov chain, but not on the size of the portfolio at the claim time.

Motivated by various applications in communications systems, there have recently been several studies of fluid systems that alternate between 'on-periods' and 'off-periods'. During off-periods, the buffer content increases in some state-dependent way, and during on-periods it decreases at a state-dependent rate (unless the on-period starts at 0). In [10] the off-periods were exponentially distributed. In [11] and [23] the rate at which the system switches from on to off (and back) depends on the actual buffer content. It should be noted that if the on-period is compressed to be of zero duration and the state-dependent decrease rate goes to infinity in an appropriate way, a process in which the size of the downward jump depends on the buffer content could be obtained.

Altman et al. [4] studied GCPs in relation to the transmission control protocol of the Internet. Variants of the transmission control protocol lead to adaptive window protocols under which the window size alternately grows for a certain period and decreases instantaneously according to some function of the present window size. For example, under the additive-increasemultiplicative-decrease protocol the window size grows linearly, but at jump epochs decreases in proportion to its present size. For a quite general adaptive window protocol, Altman et al. [4] obtained stability conditions and derived the steady-state window size distribution in analytic form. See [3], [12], and [19] for studies of the additive-increase-multiplicativedecrease protocol. As will be shown in Section 5, there is a one-to-one correspondence between a particular GCP and the well-known shot noise process. This particular GCP corresponds to the additive-increase-multiplicative-decrease rule, except that the decrements at jump epochs are not deterministically, but rather stochastically, proportional to the level before the jump (for example being uniformly distributed between 0 and that level).

\section{GCPs as piecewise-deterministic Markov processes}

Piecewise-deterministic Markov processes in general were analyzed by Davis [14], [15, Sections 1-3]; also see the presentation in [22, pp. 444-482]. We will specialize this theory to the growth-collapse processes defined by (i)-(iii) above. This yields the following key results. 
Proposition 1. The infinitesimal generator of $X$ is given by

$$
(g f)(x)=f^{\prime}(x)+r(x) \int_{[0, x)}(f(y)-f(x)) \mu_{x}(\mathrm{~d} y),
$$

where a prime denotes differentiation. Its domain, $\mathcal{D}(\mathcal{G})$, contains all functions $f: \mathbb{R}_{+} \rightarrow \mathbb{R}$ that are locally bounded and absolutely continuous.

Proof. This follows from Theorem 11.2.2 of [22].

In the following we denote by $\mathrm{P}_{x}(\cdot)$ and $\mathrm{E}_{x}(\cdot)$ the conditional probability and conditional expectation given that $X_{0}=x$, respectively, and by $\lfloor x\rfloor$ the integer part of $x$.

Proposition 2. The hitting times $T_{a}$ satisfy

$$
\mathrm{E}_{x}\left(T_{a}\right) \leq \mathrm{E}_{0}\left(T_{a}\right)<\infty \text { for all } a>x .
$$

Proof. The first inequality is obvious. Regarding the integrability of $T_{a}$ under $\mathrm{P}_{0}$, observe that $T_{a}>t$ implies that there occurs at least one jump in each of the time intervals $(0, a],(a, 2 a], \ldots,((\lfloor t / a\rfloor-1) a,\lfloor t / a\rfloor a]$ if $t \geq a$, and that $\mathrm{P}_{0}\left(T_{a}>t\right)=1$ for $t<a$. Indeed, if $T_{a}>(k-1) a$ and there are no jumps in $((k-1) a, k a]$ for some $k \in\{1, \ldots,\lfloor t / a\rfloor\}$, then $0 \leq X_{(k-1) a}<a$ and $X_{k a}=X_{(k-1) a}+a>a$; thus, $X_{t}=a$ for some $t \in((k-1) a, k a)$. It follows that

$$
\begin{aligned}
\mathrm{P}_{0}\left(T_{a}>t\right) & \leq\left(1-\exp \left\{-\int_{0}^{a} r(s) \mathrm{d} s\right\}\right)\left(1-\exp \left\{-\min _{0 \leq x \leq a} \int_{x}^{x+a} r(s) \mathrm{d} s\right\}\right)^{\lfloor t / a\rfloor-1} \\
& \leq\left(1-r_{a}\right)^{\lfloor t / a\rfloor}
\end{aligned}
$$

where

$$
r_{a}=\exp \left\{-\min _{0 \leq x \leq a} \int_{x}^{x+a} r(s) \mathrm{d} s\right\}
$$

Thus, $r_{a} \in(0,1)$ and

$$
\mathrm{E}_{0}\left(T_{a}\right)=\int_{0}^{\infty} \mathrm{P}_{0}\left(T_{a}>t\right) \mathrm{d} t \leq \int_{0}^{\infty}\left(1-r_{a}\right)^{t / a} \mathrm{~d} t=\frac{a}{\left|\log \left(1-r_{a}\right)\right|}
$$

Proposition 3. Define $\rho(x)=r(x) \int_{0}^{x} \mu_{x}([0, u)) \mathrm{d} u$. If, for some $\varepsilon>0$ and some $a>0$,

$$
\rho(x)>1+\varepsilon \text { for all } x \geq a,
$$

then

$$
\mathrm{E}_{x}\left(T_{a}\right)<\infty \text { for all } x \geq a \text {. }
$$

Proof. Let $i(x)=x$ be the identity function and note that, by Dynkin's formula, the process

$$
X_{t \wedge T_{a}}-\int_{0}^{t \wedge T_{a}}\left(g_{i}\right)\left(X_{s}\right) \mathrm{d} s
$$

is a $\mathrm{P}_{x}$-martingale for every $x \geq a$. By Proposition 1 , we have $(g i)(x)=1-\rho(x)$, and (2) and the definition of $T_{a}$ thus yield

$$
\begin{aligned}
x & =\mathrm{E}_{x}\left(X_{0}\right) \\
& =\mathrm{E}_{x}\left(X_{t \wedge T_{a}}-\int_{0}^{t \wedge T_{a}}\left(1-\rho\left(X_{S}\right)\right) \mathrm{d} s\right) \\
& \geq a+\varepsilon \mathrm{E}_{x}\left(t \wedge T_{a}\right) \quad \text { for all } x \geq a .
\end{aligned}
$$

Letting $t \rightarrow \infty$ leads to $\mathrm{E}_{x}\left(T_{a}\right) \leq(x-a) / \varepsilon$. 
Proposition 4. If $Q$ is a stationary distribution of $X$, then $Q$ has a density $q(x)$ satisfying the integral equation

$$
q(x)=\int_{x}^{\infty} r(t) \mu_{t}([0, x)) q(t) \mathrm{d} t .
$$

Proof. It follows from [14] that if $Q$ is a stationary distribution, then

$$
\int\left(g f_{s}\right)(x) \mathrm{d} Q(x)=0 \quad \text { for all } s>0,
$$

where $f_{s}(x)=\mathrm{e}^{-s x}$. Substituting using (1) and integrating by parts yields

$$
\int_{0}^{\infty}\left(f_{s}^{\prime}(x)-r(x) \int_{0}^{x} f_{s}^{\prime}(u) \mu_{x}([0, u)) \mathrm{d} u\right) \mathrm{d} Q(x)=0,
$$

which is tantamount to

$$
\int_{0}^{\infty} \mathrm{e}^{-s x} \mathrm{~d} Q(x)=\int_{0}^{\infty} \mathrm{e}^{-s u}\left(\int_{u-}^{\infty} r(x) \mu_{x}([0, u)) \mathrm{d} Q(x)\right) \mathrm{d} u
$$

for all $s>0$. It follows that $Q$ has a density $q$ satisfying (3).

Proposition 5. If

$$
\liminf _{x \rightarrow \infty} \rho(x)>1
$$

then $X$ has exactly one stationary distribution $Q$, and $X_{t} \rightarrow Q$ weakly as $t \rightarrow \infty$.

Proof. Since the expected recurrence times for any $a>0$ are finite, by Propositions 2 and 3 , this follows from the ergodic theorem for regenerative processes [6, p. 170].

Now let us turn to the hitting time $T_{a}$. To determine its Laplace transform, $\mathrm{E}_{x}\left(\mathrm{e}^{-\alpha T_{a}}\right)$, in the sequel we use the method presented in [18], which requires us to find a solution $f_{\alpha}$ to the equation

$$
\alpha f(x)=(g f)(x)
$$

that is positive and bounded on $[0, a]$, for any $\alpha>0$. Then, by [18], the LT is simply given by

$$
\mathrm{E}_{x}\left(\mathrm{e}^{-\alpha T_{a}}\right)=\frac{f_{\alpha}(x)}{f_{\alpha}(a)}, \quad 0 \leq x<a .
$$

Example 1. Let $\mu_{x}(\mathrm{~d} y)$ have a probability density of the form

$$
p(x, y)=a(x) b(y) 1_{(0, x)}(y),
$$

for certain Borel-measurable functions $a:(0, \infty) \rightarrow[0, \infty)$ and $b:(0, \infty) \rightarrow[0, \infty)$. In particular, this covers the case $p(x, y)=x^{-1} 1_{(0, x)}(y)$, in which at every crash a uniformly distributed piece of $X_{t-}$ is 'cut off'.

With $B(x)=\int_{0}^{x} b(y) \mathrm{d} y$, we have $a(x)=1 / B(x)$ and $\mu_{t}([0, x))=B(x) / B(t)$. By Proposition $5, X$ has a stationary distribution if

$$
\liminf _{x \rightarrow \infty} \frac{r(x)}{B(x)} \int_{0}^{x} B(u) \mathrm{d} u>1 .
$$


By Proposition 4, for the stationary density we obtain the integral equation

$$
q(x)=B(x) \int_{x}^{\infty} \frac{r(t)}{B(t)} q(t) \mathrm{d} t,
$$

which is easily solved:

$$
q(x)=C B(x) \exp \left\{-\int_{0}^{x} r(u) \mathrm{d} u\right\}
$$

where the normalizing constant $C$ is given by

$$
C=\left(\int_{0}^{\infty} B(x) \exp \left\{-\int_{0}^{x} r(u) \mathrm{d} u\right\} \mathrm{d} x\right)^{-1} .
$$

To find the LT of $T_{a}$ we have to solve the integral equation

$$
\alpha f(x)=f^{\prime}(x)-\frac{r(x)}{B(x)} \int_{0}^{x} f^{\prime}(u) B(u) \mathrm{d} u .
$$

Assuming that $r(x)$ is differentiable, we obtain from (6) the second-order differential equation

$$
f^{\prime \prime}(x)-[\alpha+r(x)+g(x)] f^{\prime}(x)+\alpha g(x) f(x)=0 .
$$

Below we will find the suitable solution in some special cases.

Example 2. Let $r(x) \equiv \lambda$ be constant. Condition (4), sufficient for the existence and uniqueness of a stationary distribution, becomes

$$
\liminf _{x \rightarrow \infty} \mathrm{E}\left(J_{x}\right)>\lambda^{-1}
$$

where $J_{x}$ is the size of a generic jump starting from level $x$. Formula (3) for the stationary density transforms into

$$
q(x)=\lambda \int_{x}^{\infty} \mu_{t}([0, x)) q(t) \mathrm{d} t .
$$

This integral equation can be solved in terms of a Neumann series.

Regarding the LT of $T_{a}$, (1) becomes

$$
(\alpha+\lambda) f(x)=f^{\prime}(x)+\lambda \int_{[0, x)} f(y) \mu_{x}(\mathrm{~d} y) .
$$

If $\mu_{x}$ has a density $p(x, y)$ on $(0, x)$ and an atom of mass $p_{0}(x)$ at 0 , we obtain

$$
(\alpha+\lambda) f(x)=f^{\prime}(x)+\lambda \int_{[0, x)} f(y) p(x, y) \mathrm{d} y+p_{0}(x) f(0) .
$$

Example 3. In the standard renewal age process we have $\mu_{x}=\varepsilon_{0}$, the point mass at 0 . The well-known stationary density $q(x)=\mathrm{P}_{0}(\sigma>x) / \mathrm{E}_{0}(\sigma)$ can be easily derived from (3). Formula (5) takes the form

$$
\alpha f(x)=f^{\prime}(x)+r(x)(f(0)-f(x)),
$$


a first-order linear differential equation that can be easily solved explicitly. Let $R(x)=$ $\int_{0}^{x} r(u) \mathrm{d} u$. The function

$$
f_{\alpha}(x)=\mathrm{e}^{\alpha x+R(x)}\left[1-\int_{0}^{x} r(u) \mathrm{e}^{-\alpha u-R(u)} \mathrm{d} u\right]
$$

is a positive solution to (7) such that the LT of $T_{a}$ is given by

$$
\mathrm{E}\left(\mathrm{e}^{-\alpha T_{a}}\right)=\frac{\exp \{\alpha(x-a)+R(x)-R(a)\}\left[1-\int_{0}^{x} r(u) \mathrm{e}^{-\alpha u-R(u)} \mathrm{d} u\right]}{1-\int_{0}^{a} r(u) \mathrm{e}^{-\alpha u-R(u)} \mathrm{d} u} .
$$

Example 4. Let $\mu_{x}=\varepsilon_{c(x)}$, for some strictly increasing continuous function $c:(0, \infty) \rightarrow$ $(0, \infty)$ satisfying $c(x)<x$ for all $x>0$. This is the situation in additive-increase-multiplicative-decrease window protocols, as studied in [4]. By Propositions 4 and 5, $X$ has a stationary distribution if $\lim _{\inf } \rightarrow \infty r(x)(x-c(x))>1$, and if this holds then the stationary density $q(x)$ satisfies

$$
q(x)=\int_{x}^{c^{-1}(x)} r(t) q(t) \mathrm{d} t,
$$

an equation that does not easily admit closed-form solutions. However, in the special case in which $r(x) \equiv \lambda$ and $c(x)=c x$ for some constant $c \in(0,1)$, the stationary distribution has a simple representation. Let $X$ be in steady state. Then, if $\sigma_{1}$ and $\sigma_{2}$ denote the times of the first and second jumps, respectively, it follows from the PASTA ('Poisson Arrivals See Time Averages') property that the left-hand limits $X_{\sigma_{1}-}$ and $X_{\sigma_{2}-}$ both have the stationary distribution. Next, note that $X_{\sigma_{2}-}=c X_{\sigma_{1}-}+Y$, where $Y$ is $\operatorname{Exp}(\lambda)$-distributed and independent of $X_{\sigma_{1}-}$. Now let $X_{\mathrm{e}}$ be a random variable having the stationary distribution and let $Y_{1}, Y_{2}, \ldots$ be i.i.d., $\operatorname{Exp}(\lambda)$-distributed, and independent of $X_{\mathrm{e}}$. Then

$$
X_{\mathrm{e}} \stackrel{\mathrm{D}}{=} c X_{\mathrm{e}}+Y_{1} \stackrel{\mathrm{D}}{=} c^{2} X_{\mathrm{e}}+c Y_{2}+Y_{1} \stackrel{\mathrm{D}}{=} \cdots .
$$

It follows that the stationary distribution of $X$ is equal to that of $\sum_{i=0}^{\infty} c^{i} Y_{i}$.

\section{Uniform cut-offs and proportional jump intensity}

A very nice special case of Example 1 is the following:

(a) $p(x, y)=x^{-1} 1_{(0, x)}(y)$;

(b) $r(x)=\lambda x$.

Thus, the jump rate at time $t$ is proportional to $X_{t-}$ and at a jump time a uniformly distributed piece of $X_{t-}$ is cut off. In this case the generator is of the form

$$
(g f)(x)=f^{\prime}(x)+\lambda \int_{[0, x)}(f(y)-f(x)) \mathrm{d} y .
$$

Propositions 4 and 5 immediately yield the following theorem.

Theorem 1. The stationary density of $X$ and the LT of $T_{a}$ are respectively given by

$$
\begin{aligned}
q(x) & =\lambda x \mathrm{e}^{-\lambda x^{2} / 2} \text { and } \\
\mathrm{E}_{x}\left(\mathrm{e}^{-\alpha T_{a}}\right) & =\frac{1+\alpha \int_{0}^{x} \mathrm{e}^{\alpha y+\left(\lambda y^{2} / 2\right)} \mathrm{d} y}{1+\alpha \int_{0}^{a} \mathrm{e}^{\alpha y+\left(\lambda y^{2} / 2\right)} \mathrm{d} y},
\end{aligned}
$$

for $0 \leq x<a$. 
Proof. By (3), the stationary density of $X$ satisfies

$$
q(x)=\int_{x}^{\infty} \lambda t\left(\frac{x}{t}\right) q(t) \mathrm{d} t=\lambda x \int_{x}^{\infty} q(t) \mathrm{d} t,
$$

and the right-hand side of (8) is the only density solving (10).

Formula (5) becomes

$$
\alpha f(x)=f^{\prime}(x)+\lambda \int_{0}^{x} f(y) \mathrm{d} y-\lambda x f(x) .
$$

Taking the derivative in (11) yields

$$
\alpha f^{\prime}(x)=f^{\prime \prime}(x)-\lambda x f^{\prime}(x)
$$

It follows that $f$ is of the form

$$
f(x)=C+D \int_{0}^{x} \mathrm{e}^{\alpha y+\left(\lambda y^{2} / 2\right)} \mathrm{d} y .
$$

To find $\mathrm{E}_{x}\left(\mathrm{e}^{-\alpha T_{a}}\right)$ we may assume that $f(0)=1$ (in view of (5)), i.e. $C=1$. It follows from (12) that $\alpha f(0)=f^{\prime}(0)$, whence $f^{\prime}(0)=\alpha$. Hence, $D=1$ and, thus, (13) is equal to the numerator of (9) and provides a solution, $f_{\alpha}(x)$, to (5) that is positive and bounded on $[0, a]$. Formula (9) now follows from (5).

The following theorem describes the asymptotic behavior of $T_{a}$ as $a \rightarrow \infty$.

Theorem 2. As $a \rightarrow \infty$,

$$
\frac{\log T_{a}}{a^{2}} \rightarrow \frac{\lambda}{2} \text { in probability. }
$$

Proof. Let $M_{t}=\max _{0 \leq s \leq t} X_{s}$. We will show that

$$
(\log t)^{-1 / 2} M_{t} \stackrel{\mathrm{D}}{=}(2 / \lambda)^{1 / 2} \quad \text { as } t \rightarrow \infty
$$

Relation (15) implies (14). Indeed, let $g(t)=[(2 / \lambda) \log t]^{1 / 2}$. Then, by (15),

$$
\begin{aligned}
& \lim _{t \rightarrow \infty} \mathrm{P}_{0}\left(M_{t} \geq(1+\delta) g(t)\right)=0, \\
& \lim _{t \rightarrow \infty} \mathrm{P}_{0}\left(M_{t} \geq(1-\delta) g(t)\right)=1,
\end{aligned}
$$

for every $\delta>0$. Because $T_{a} \leq t$ if and only if $M_{t} \geq a$, (16) and (17) are tantamount to

$$
\begin{aligned}
& \lim _{t \rightarrow \infty} \mathrm{P}_{0}\left(T_{(1+\delta) g(t)} \leq t\right)=0, \\
& \lim _{t \rightarrow \infty} \mathrm{P}_{0}\left(T_{(1-\delta) g(t)} \leq t\right)=1 .
\end{aligned}
$$

By first letting $a=(1-\delta) g(t)$ and then letting $a=(1+\delta) g(t)$, from (18) and (19) we obtain

$$
\begin{aligned}
& \lim _{a \rightarrow \infty} \mathrm{P}_{0}\left(T_{a} \leq \exp \left\{(1+\varepsilon)(\lambda / 2) a^{2}\right\}\right)=0, \\
& \lim _{a \rightarrow \infty} \mathrm{P}_{0}\left(T_{a} \leq \exp \left\{(1-\varepsilon)(\lambda / 2) a^{2}\right\}\right)=1,
\end{aligned}
$$


for all $\varepsilon>0$. Relations (20) and (21) immediately yield (14). Thus, it is sufficient to prove (15). We will now actually show that

$$
\lim _{t \rightarrow \infty} \mathrm{E}_{0}\left(\left([(2 / \lambda) \log t]^{-1 / 2} M_{t}\right)^{n}\right)=1 \quad \text { for all } n \in \mathbb{N} .
$$

By (22), all moments of $[(2 / \lambda) \log t]^{-1 / 2} M_{t}$ converge to 1 . This implies (15).

We know from (9) that

$$
\mathrm{E}_{0}\left(\mathrm{e}^{-\alpha T_{a}}\right)=\left(1+\alpha \int_{0}^{a} \mathrm{e}^{\alpha y+\left(\lambda y^{2} / 2\right)} \mathrm{d} y\right)^{-1}
$$

Let $v$ be the measure on $[0, \infty)$ defined by $v([0, t])=\mathrm{E}_{0}\left(M_{t}^{n}\right)$. Because $\left\{T_{a} \leq t\right\}=\left\{M_{t} \geq a\right\}$, we have

$$
\begin{aligned}
\int_{0}^{\infty} \mathrm{e}^{-\alpha t} \mathrm{~d} v(t) & =\int_{0}^{\infty} \mathrm{e}^{-\alpha t} \frac{\mathrm{d}}{\mathrm{d} t} \mathrm{E}_{0}\left(M_{t}^{n}\right) \mathrm{d} t \\
& =\int_{0}^{\infty} \mathrm{e}^{-\alpha t}\left(\frac{\mathrm{d}}{\mathrm{d} t} \int_{0}^{\infty} n a^{n-1} \mathrm{P}_{0}\left(M_{t} \geq a\right) \mathrm{d} a\right) \mathrm{d} t \\
& =\int_{0}^{\infty} \int_{0}^{\infty} n a^{n-1} \mathrm{e}^{-\alpha t} \mathrm{P}_{0}\left(T_{a} \in \mathrm{d} t\right) \mathrm{d} a \\
& =n \int_{0}^{\infty} a^{n-1} \mathrm{E}_{0}\left(\mathrm{e}^{-\alpha T_{a}}\right) \mathrm{d} a \\
& =n \int_{0}^{\infty} a^{n-1}\left(1+\alpha \int_{0}^{a} \mathrm{e}^{\alpha y+\left(\lambda y^{2} / 2\right)} \mathrm{d} y\right)^{-1} \mathrm{~d} a
\end{aligned}
$$

We now prove that

$$
\lim _{\alpha \searrow 0}\left(\frac{2}{\lambda}|\log \alpha|\right)^{-n / 2} \int_{0}^{\infty} \mathrm{e}^{-\alpha t} \mathrm{~d} \nu(t)=1 \quad \text { for all } n \in \mathbb{N} .
$$

As $|\log \alpha|$ is slowly varying at 0 , the standard Tauberian theorem yields

$$
\lim _{t \rightarrow \infty}\left(\frac{2}{\lambda}|\log (1 / t)|\right)^{-n / 2} v([0, t])=1 \quad \text { for all } n \in \mathbb{N},
$$

which is what we desired to show.

Of course, we have to consider the right-hand integral in (23) as $\alpha \rightarrow 0$. Its integrand tends to the nonintegrable $a^{n-1}$, which is why we have to introduce an appropriate normalizing factor. Denote the right-hand side of (23) by $H_{n}(\alpha)$. We have to estimate $H_{n}(\alpha)$ from below and from above. For any $b>0$, we have

$$
1+\alpha \int_{0}^{a} \mathrm{e}^{\alpha y+\left(\lambda y^{2} / 2\right)} \mathrm{d} y \leq 1+\alpha b \mathrm{e}^{\alpha b+\left(\lambda b^{2} / 2\right)} \text { for all } a \in[0, b] .
$$

Hence,

$$
\begin{aligned}
H_{n}(\alpha) & \geq n \int_{0}^{b} a^{n-1}\left(1+\int_{0}^{a} \mathrm{e}^{\alpha y+\left(\lambda y^{2}\right) / 2} \mathrm{~d} y\right)^{-1} \mathrm{~d} a \\
& \geq \frac{b^{n}}{1+\alpha b \mathrm{e}^{\alpha b+\left(\lambda b^{2} / 2\right)}} \quad \text { for all } b>0
\end{aligned}
$$


Let $\delta \in(0,1)$ and set $b \equiv b(\delta, \alpha)=[(1-\delta)(2 / \lambda)|\log \alpha|]^{1 / 2}$ in (25). It follows that

$$
H_{n}(\alpha) b(\delta, \alpha)^{-n} \geq\left[1+\alpha^{\delta} b(\delta, \alpha) \mathrm{e}^{\alpha b(\delta, \alpha)}\right]^{-1} .
$$

Because $\lim _{\alpha \searrow 0} \alpha^{\delta} b(\delta, \alpha)=0,(26)$ yields

$$
\liminf _{\alpha \searrow 0}(1-\delta)^{-n / 2}\left(\frac{2}{\lambda}|\log \alpha|\right)^{-n / 2} H_{n}(\alpha) \geq 1
$$

for all $\delta \in(0,1)$ and all $n \in \mathbb{N}$. Thus,

$$
\liminf _{\alpha \searrow 0}\left(\frac{2}{\lambda}|\log \alpha|\right)^{-n / 2} H_{n}(\alpha) \geq 1 .
$$

To find an upper bound, we use

$$
\begin{aligned}
\int_{0}^{a} \mathrm{e}^{\alpha y+\left(\lambda y^{2}\right) / 2} \mathrm{~d} y & \geq \int_{0}^{a} \mathrm{e}^{\lambda y^{2} / 2} \mathrm{~d} y \\
& \geq \int_{a-1}^{a} \mathrm{e}^{\lambda y^{2} / 2} \mathrm{~d} y \\
& \geq \mathrm{e}^{\lambda(a-1)^{2} / 2},
\end{aligned}
$$

which holds for all $a \geq 1$. Hence, for any $b>0$,

$$
\begin{aligned}
H_{n}(\alpha) & \leq \int_{0}^{b+1} a^{n-1} \mathrm{~d} a+\alpha^{-1} n \int_{b+1}^{\infty} a^{n-1} \mathrm{e}^{-\lambda(a-1)^{2} / 2} \mathrm{~d} a \\
& \leq(b+1)^{n}+\alpha^{-1} n \int_{b+1}^{\infty}\left(\frac{b+1}{b}(a-1)\right)^{n-1} \mathrm{e}^{-\lambda(a-1)^{2} / 2} \mathrm{~d} a \\
& =(b+1)^{n}+\alpha^{-1} n\left(1+b^{-1}\right)^{n-1} \lambda^{-(n-1) / 2} \int_{\lambda^{1 / 2} b}^{\infty} x^{n-1} \mathrm{e}^{-x^{2} / 2} \mathrm{~d} x
\end{aligned}
$$

The integral on the right-hand side of (28) is bounded as follows: for every $N \in \mathbb{N}$, there is a constant $C_{N}>0$ such that

$$
\int_{t}^{\infty} x^{N} \mathrm{e}^{-x^{2} / 2} \mathrm{~d} x \leq C_{N} t^{N-1} \mathrm{e}^{-t^{2} / 2} \text { for all } t>0 .
$$

By (28) and (29),

$$
H_{n}(\alpha) \leq(b+1)^{n}+\alpha^{-1} n\left(1+b^{-1}\right)^{n-1} \lambda^{-(n-1) / 2} C_{n-1}\left(\lambda^{1 / 2} b\right)^{n-2} \mathrm{e}^{-\lambda b^{2} / 2}
$$

for all $b>0$. Now set $b \equiv b(\alpha)=[(2 / \lambda)|\log \alpha|]^{1 / 2}$. From (30) we conclude that

$$
\begin{aligned}
b(\alpha)^{-n} H_{n}(\alpha) & \leq\left(1+b(\alpha)^{-1}\right)^{n}+n C_{n-1}\left(1+b(\alpha)^{-1}\right)^{n-1} \lambda^{-1 / 2} \alpha^{-1} b(\alpha)^{-2} \mathrm{e}^{-\lambda b(\alpha)^{2} / 2} \\
& =\left(1+b(\alpha)^{-1}\right)^{n-1}\left[1+b(\alpha)^{-1}+\frac{1}{2} n C_{n-1} \lambda^{1 / 2}|\log \alpha|^{-2}\right] \\
& \rightarrow 1 \quad \text { as } \alpha \searrow 0 .
\end{aligned}
$$

Thus, $\lim \sup _{\alpha \searrow 0} b(\alpha)^{-n} H_{n}(\alpha) \leq 1$, which, together with (27), yields

$$
\lim _{\alpha \searrow 0} b(\alpha)^{-n} H_{n}(\alpha)=1
$$

for every $n \in \mathbb{N}$. This is equivalent to (24). 


\section{Poisson jump times with uniform cut-offs}

We now consider the case in which

(a) $p(x, y)=x^{-1} 1_{(0, x)}(y)$;

(b) $r(x) \equiv \lambda>0$.

Thus, jumps arrive at Poisson times with intensity $\lambda$ and the cut-off mechanism is the same as in Section 3.

Theorem 3. The stationary distribution of $X$ is $\operatorname{Erlang}(2, \lambda)$, i.e. has density

$$
q(x)=\lambda^{2} x \mathrm{e}^{-\lambda x} \text {. }
$$

The LT of $T_{a}$ is given by $\mathrm{E}_{x}\left(\mathrm{e}^{-\alpha T_{a}}\right)=f_{\alpha}(x) / f_{\alpha}(a), 0 \leq x<a$, where $f_{a}(x)$ is the unique solution to the differential equation

$$
x f^{\prime \prime}(x)+(1-(\lambda+\alpha) x) f^{\prime}(x)-\alpha f(x)=0, \quad x \geq 0,
$$

with initial conditions

$$
f(0)=1, \quad f^{\prime}(0)=\alpha .
$$

Proof. By (3), the stationary density of $X$ satisfies

$$
q(x)=\int_{x}^{\infty} \lambda\left(\frac{x}{t}\right) q(t) \mathrm{d} t=\lambda x \int_{x}^{\infty} \frac{q(t)}{t} \mathrm{~d} t .
$$

The right-hand side of (31) is the only density solving (34).

Formula (5) becomes

$$
\alpha f(x)=f^{\prime}(x)+\frac{\lambda}{x} \int_{0}^{x} f(y) \mathrm{d} y-\lambda f(x) .
$$

Multiplying by $x$ and taking the derivative in (35) yields (32). The initial condition $f(0)=1$ can be fixed arbitrarily, and the condition $f^{\prime}(0)=\alpha$ then follows by letting $x$ tend to 0 in (35).

Formula (32) is a variant of the degenerate hypergeometric differential equation. Its general solution is given by

$$
f(x)=\mathrm{e}^{(\lambda+\alpha) x}\left[C_{1} \Phi\left(\frac{\lambda}{2(\lambda+\alpha)}, 1 ;-2(\lambda+\alpha) x\right)+C_{2} \Psi\left(\frac{\lambda}{2(\lambda+\alpha)}, 1 ;-2(\lambda+\alpha) x\right)\right] .
$$

Here

$$
\Phi(a, b ; x)=1+\sum_{k=1}^{\infty} \frac{(a)_{k}}{(b)_{k}} \frac{x^{k}}{k !}
$$

is Kummer's series (in which $(a)_{k}=a(a+1) \cdots(a+k-1)$, with $\left.(a)_{0}=1\right)$ and

$$
\begin{aligned}
& \Psi(a, 1 ; x) \\
& =\frac{1}{\Gamma(a-1)}\left\{\Phi(a, 2 ; x) \log x+\sum_{r=0}^{\infty}[\psi(a+r)-\psi(1+r)-\psi(2+r)] \frac{(a)_{r} x^{r}}{r !(r+1) !}\right\} \\
& \quad+\frac{1}{\Gamma(a)},
\end{aligned}
$$


where $\psi(z)=\Gamma^{\prime}(z) / \Gamma(z)$ is the logarithmic derivative of the gamma function (see [21, Equations 2.1.2.103 and 2.1.2.65]). In our case we can also use the formula

$$
\Phi(a, 1 ; x)=\frac{1}{\Gamma(a) \Gamma(1-a)} \int_{0}^{1} \mathrm{e}^{t x} t^{a-1}(1-t)^{-a} \mathrm{~d} t, \quad 0<a<1 .
$$

The constants $C_{1}$ and $C_{2}$ are uniquely determined by the initial conditions (33), which become transcendental equations involving the functions $\Phi$ and $\Psi$.

Next, we derive the expected value and the variance of $X_{t}$. Let $X_{0}=0$. We can write $X_{t}$ in the form

$$
X_{t}=t-\sum_{n=0}^{N(t)} \tau_{n} W_{n}^{(N(t))}
$$

Here $(N(t))_{t \geq 0}$ is a homogeneous Poisson counting process with rate $\lambda, \tau_{0} \equiv 0$, and $\tau_{1}, \tau_{2}, \ldots$, with $0<\tau_{1}<\tau_{2}<\cdots$, denote the jump times of $(N(t))_{t \geq 0}$. Furthermore, $W_{0}^{(m)} \equiv 1$ and

$$
W_{n}^{(m)}=1(n=m)\left(1-U_{m}\right)+1(n<m)\left(1-U_{n}\right) \prod_{j=n+1}^{m} U_{j}, \quad n \geq 1,
$$

where $U_{1}, U_{2}, \ldots$ are i.i.d. random variables that are independent of $\{N(t), t \geq 0\}$ and have the uniform distribution on $(0,1)$. By $1(A)$ we denote the indicator function of the event $A$.

Let $Y_{t}=t-X_{t}$. Clearly, $Y_{t}$ is the sum of the sizes of all jumps in $[0, t]$.

Theorem 4. For every $t \in[0, \infty)$,

$$
\mathrm{E}\left(X_{t}\right)=\frac{2}{\lambda}\left(1-\mathrm{e}^{-\lambda t / 2}\right)
$$

Proof. We have

$$
\begin{aligned}
\mathrm{E}\left(X_{t}\right) & =t-\mathrm{E}\left(Y_{t}\right) \\
& =t-\mathrm{E}\left(\mathrm{E}\left(Y_{t} \mid N(t)=n\right)\right) .
\end{aligned}
$$

Obviously, $\mathrm{E}\left(Y_{t} \mid N(t)=0\right)=0$. For $n \geq 1$, it is well known that the conditional distribution of $\tau_{i}$, given that $N(t)=n$, is $\operatorname{Beta}(i, n+1-i)$. Accordingly, due to the independence of $\tau_{i}$ and $W_{i}^{(n)}$, for all $n \geq 1$ we obtain

$$
\begin{aligned}
\mathrm{E}\left(Y_{t} \mid N(t)=n\right) & =\mathrm{E}\left(\sum_{i=1}^{n} \tau_{i} W_{i}^{(n)} \mid N(t)=n\right) \\
& =t \sum_{i=1}^{n} \frac{i}{n+1}\left(\frac{1}{2}\right)^{n+1-i} \\
& =t\left(1-\frac{2}{n+1}\left(1-\left(\frac{1}{2}\right)^{n+1}\right)\right) .
\end{aligned}
$$


With $p(n ; \lambda t)=\mathrm{e}^{-\lambda t}(\lambda t)^{n} / n !, n=0,1, \ldots$, we find that

$$
\begin{aligned}
\mathrm{E}\left(X_{t}\right) & =t-\mathrm{E}\left(Y_{t} \mid N(t)\right) \\
& =2 t \mathrm{E}\left(\frac{1}{N(t)+1}\left(1-\left(\frac{1}{2}\right)^{N(t)+1}\right)\right) \\
& =2 t \sum_{n=0}^{\infty} p(n ; \lambda t) \frac{1}{n+1}\left(1-\left(\frac{1}{2}\right)^{n+1}\right) \\
& =\frac{2}{\lambda} \sum_{n=1}^{\infty} p(n ; \lambda t)\left(1-\left(\frac{1}{2}\right)^{n}\right) .
\end{aligned}
$$

Formula (36) is easily obtained from (38).

Theorem 5. For fixed the variance of $X_{t}$ is

$$
\operatorname{var}\left\{X_{t}\right\}=\frac{1}{\lambda^{2}}\left(2-4 \mathrm{e}^{-\lambda t}-16 \mathrm{e}^{-\lambda t / 2}+18 \mathrm{e}^{-2 \lambda t / 3}\right) .
$$

Proof. We have

$$
\begin{aligned}
\operatorname{var}\left\{X_{t}\right\} & =\operatorname{var}\left\{Y_{t}\right\} \\
& =\operatorname{var}\left\{\sum_{n=0}^{N(t)} \tau_{n} W_{n}^{(N(t))}\right\} \\
& =\mathrm{E}\left(\operatorname{var}\left\{\sum_{n=0}^{N(t)} \tau_{n} W_{n}^{(N(t))} \mid N(t)\right\}\right)+\operatorname{var}\left\{\mathrm{E}\left(\sum_{n=0}^{N(t)} \tau_{n} W_{n}^{(N(t))} \mid N(t)\right)\right\} .
\end{aligned}
$$

Since $\operatorname{var}\left\{\mathrm{E}\left(Y_{t} \mid N(t)\right)\right\}=\operatorname{var}\left\{\mathrm{E}\left(X_{t} \mid N(t)\right)\right\}$, we find from (37) that the second term on the right-hand side of (40) is

$$
\begin{aligned}
\operatorname{var}\left\{\mathrm{E}\left(X_{t} \mid N(t)\right)\right\} & =4 t^{2} \operatorname{var}\left\{\frac{1}{N(t)+1}\left(1-\left(\frac{1}{2}\right)^{N(t)+1}\right)\right\} \\
& =4 t^{2} \sum_{n=0}^{\infty} p(n ; \lambda t)\left[\frac{1}{(n+1)^{2}}\left(1-\left(\frac{1}{2}\right)^{n+1}\right)^{2}\right]-\frac{4}{\lambda^{2}}\left(1-\mathrm{e}^{-\lambda t / 2}\right)^{2}
\end{aligned}
$$

Regarding the first term on the right-hand side of (40), we notice that

$$
\operatorname{var}\left\{\sum_{n=0}^{N(t)} \tau_{n} W_{n}^{(N(t))} \mid N(t)=0\right\}=0
$$

and, for $n \geq 1$,

$$
\begin{aligned}
\operatorname{var}\left\{\sum_{i=1}^{n} \tau_{i} W_{i}^{(n)} \mid N(t)=n\right\}= & \operatorname{var}\left\{\mathrm{E}\left(\sum_{i=1}^{n} \tau_{i} W_{i}^{(n)} \mid N(T)=n, \boldsymbol{W}^{(n)}\right) \mid N(t)=n\right\} \\
& +\mathrm{E}\left(\operatorname{var}\left\{\sum_{i=1}^{n} \tau_{i} W_{i}^{(n)} \mid N(t)=n, \boldsymbol{W}^{(n)}\right\} \mid N(t)=n\right) .
\end{aligned}
$$


Furthermore, due to the independence of $\boldsymbol{W}^{(n)}$ and $N(t)$, we have

$$
\begin{aligned}
\operatorname{var}\left\{\mathrm{E}\left(\sum_{i=1}^{n} \tau_{i} W_{i}^{(n)} \mid N(t)=n, \boldsymbol{W}^{(n)}\right) \mid N(t)=n\right\} \\
=\operatorname{var}\left\{\frac{t}{n+1} \sum_{i=1}^{n} W_{i}^{(n)}\right\} \\
=\frac{t^{2}}{(n+1)^{2}}\left[\sum_{i=1}^{n}(n+1-i)^{2}\left(\left(\frac{1}{3}\right)^{i}-\left(\frac{1}{4}\right)^{i}\right)\right. \\
\left.\quad+2 \sum_{i=1}^{n-1} i \sum_{j=i+1}^{n} j\left(\frac{1}{2}\right)^{j-i}\left(\frac{1}{6}\left(\frac{1}{3}\right)^{n-j}-\frac{1}{4}\left(\frac{1}{4}\right)^{n-j}\right)\right] \\
=\frac{t^{2}}{2(n+1)^{2}}\left(1-4\left(\frac{1}{2}\right)^{n}+5\left(\frac{1}{3}\right)^{n}-2\left(\frac{1}{4}\right)^{n}\right) .
\end{aligned}
$$

To obtain the second term on the right-hand side of (42), we start with

$$
\begin{aligned}
\operatorname{var}\left\{\sum_{i=1}^{n} \tau_{i} W_{i}^{(n)} \mid N(t)=n, \boldsymbol{W}^{(n)}\right\}= & \sum_{i=1}^{n}\left(W_{i}^{(n)}\right)^{2} \operatorname{var}\left\{\tau_{i} \mid N(t)=n\right\} \\
& +2 \sum_{1 \leq i<j \leq n} \sum_{i} W_{i}^{(n)} W_{j}^{(n)} \operatorname{cov}\left(\tau_{i}, \tau_{j} \mid N_{t}=n\right) .
\end{aligned}
$$

Moreover,

$$
\operatorname{var}\left\{\tau_{i} \mid N(t)=n\right\}=t^{2} \frac{i(n+1-i)}{(n+1)^{2}(n+2)}, \quad i=1, \ldots, n,
$$

and, for $i<j$,

$$
\operatorname{cov}\left(\tau_{i}, \tau_{j} \mid N(t)=n\right)=t^{2} \frac{i(n+1-j)}{(n+1)^{2}(n+2)} .
$$

By substituting (45) and (46) into (44) and taking expectations, we obtain

$$
\begin{aligned}
& \mathrm{E}\left(\operatorname{var}\left\{\sum_{i=1}^{n} \tau_{i} W_{i}^{(n)} \mid N(t)=n, \boldsymbol{W}^{(n)}\right\} \mid N(t)=n\right) \\
& \quad=\frac{t^{2}}{(n+1)^{2}(n+2)}\left[\frac{n}{2}\left(3-\left(\frac{1}{3}\right)^{n}\right)-3\left(1+\left(\frac{1}{3}\right)^{n}\right)+6\left(\frac{1}{2}\right)^{n}\right] .
\end{aligned}
$$

By summing (43) and (47), for $n \geq 1$ we obtain

$$
\begin{aligned}
\operatorname{var}\left\{\sum_{i=1}^{n} \tau_{i} W_{i}^{(n)} \mid N(t)=n\right\}=\frac{t^{2}}{(n+1)^{2}(n+2)} & {\left[2 n\left(1+\left(\frac{1}{3}\right)^{n}\right)-n\left(2\left(\frac{1}{2}\right)^{n}+\left(\frac{1}{4}\right)^{n}\right)\right.} \\
& \left.-2\left(1+\left(\frac{1}{4}\right)^{n}\right)+2\left(\left(\frac{1}{3}\right)^{n}+\left(\frac{1}{2}\right)^{n}\right)\right] .
\end{aligned}
$$


Finally, (41) and (48) yield

$$
\begin{aligned}
\operatorname{var}\left\{X_{t}\right\}= & t^{2} \sum_{n=1}^{\infty} p(n ; \lambda t)\left[\frac{2(n-1)}{(n+1)^{2}(n+2)}\left(1-\left(\frac{1}{2}\right)^{n}\right)\right. \\
& \left.\quad+\frac{2}{(n+1)(n+2)}\left(\frac{1}{3}\right)^{n}-\frac{1}{(n+1)^{2}}\left(\frac{1}{4}\right)^{n}\right] \\
& +4 t^{2} \sum_{n=0}^{\infty} p(n ; \lambda t) \frac{1}{(n+1)^{2}}\left(1-\left(\frac{1}{2}\right)^{n}+\frac{1}{4}\left(\frac{1}{4}\right)^{n}\right)-\frac{4}{\lambda^{2}}\left(1-\mathrm{e}^{-\lambda t / 2}\right)^{2} \\
= & 6 t^{2} \sum_{n=1}^{\infty} p(n ; \lambda t) \frac{1}{(n+1)(n+2)}-6 t^{2} \sum_{n=1}^{\infty} p(n ; \lambda t) \frac{(1 / 2)^{n}}{(n+1)(n+2)} \\
& +2 t^{2} \sum_{n=1}^{\infty} p(n ; \lambda t) \frac{(1 / 3)^{n}}{(n+1)(n+2)}+t^{2} \mathrm{e}^{-\lambda t}-\frac{4}{\lambda^{2}}\left(1-\mathrm{e}^{-\lambda t / 2}\right)^{2} .
\end{aligned}
$$

Careful simplification of (49) yields (39).

Corollary 1. As a consequence of Theorem 5, we find that

$$
\lim _{t \rightarrow \infty} \mathrm{E}\left(X_{t}\right)=\frac{2}{\lambda} \text { and } \lim _{t \rightarrow \infty} \operatorname{var}\left\{X_{t}\right\}=\frac{2}{\lambda^{2}} .
$$

The distribution function of $Y_{t}$, for fixed $t$, is

$$
H(y ; t)= \begin{cases}\mathrm{e}^{-\lambda t}, & y=0, \\ \mathrm{e}^{-\lambda t}+\sum_{n=1}^{\infty} p(n ; \lambda t) H_{n}(y ; t), & 0<y \leq t, \\ 1, & y>t,\end{cases}
$$

where, for $n \geq 1$,

$$
H_{n}(y ; t)=\mathrm{P}\left(\sum_{i=1}^{n} \tau_{i} W_{i}^{(n)} \leq y \mid N(t)=n\right) .
$$

Denote by $h(y ; t)$ the density of $H(y ; t)$. Another important function is

$$
\mathrm{P}\left(Y_{t} \leq y \mid Y_{s}=x\right), \quad 0 \leq s<t .
$$

To compute it, we start from

$$
\begin{aligned}
\mathrm{P}\left(Y_{t}\right. & \left.\leq y \mid Y(s)=x, N(s)=m, N(t)=n, \tau_{m}\right) \\
& =\mathrm{P}\left(x W_{1}^{(n-m)}+\tau_{m} \sum_{i=1}^{n-m} W_{i}^{(n-m)}+\sum_{i=1}^{n-m} W_{i}^{(n-m)} U_{i: n-m}(t-s) \leq y\right), \\
& m \leq n, s<t,
\end{aligned}
$$

where $U_{i: n-m}(t-s)$ is the $i$ th order statistic of $n-m$ i.i.d. random variables distributed uniformly on $(0, t-s)$. As before, $W_{i}^{(n-m)}=\left(1-U_{i}\right) \prod_{j=i+1}^{n-m} U_{j}, i=1, \ldots, n-m-1$, 
and $W_{n-m}^{(n-m)}=1-U_{n-m}$, where $U_{1}, \ldots, U_{n-m}$ are i.i.d., uniformly distributed on $(0,1)$, and independent of $U_{i: n-m}(t-s), i=1, \ldots, n-m$.

The conditional distribution of $\tau_{m} / s$, given that $N(s)=m$, is $\operatorname{Beta}(m, 1)$. Also, $\tau_{m}$ is independent of $\left\{W_{i}^{(n-m)}, i=1, \ldots, n-m\right\}$ and $\left\{U_{i: n-m}(t-s), i=1, \ldots, n-m\right\}$. Hence, by $(50)$,

$$
\begin{aligned}
\mathrm{P}\left(Y_{t} \leq\right. & \left.y \mid Y_{s}=x\right) \\
= & \sum_{m=0}^{\infty} p(m ; \lambda s) \sum_{l=0}^{\infty} p(l ; \lambda(t-s)) \\
& \quad \times \frac{1}{s B(m, 1)} \int_{0}^{s} z^{m-1} \mathrm{P}\left(x W_{1}^{(l)}+z \sum_{i=1}^{l} W_{i}^{(l)}+\sum_{i=1}^{l} W_{i}^{(l)} U_{i: l}(t-s) \leq y\right) \mathrm{d} z .
\end{aligned}
$$

The probability in the integrand on the right-hand side of (51) can essentially be written as the Lebesgue measure of a $2 l$-dimensional set.

Another quantity of interest is the embedded Markov chain $X(n)=X_{\tau_{n}}$, i.e. the sequence of levels just after jumps.

Theorem 6. The stationary distribution of $X(n)$ is $\operatorname{Exp}(\lambda)$.

Proof. By the PASTA property, the levels $X_{\tau_{n}}$ just prior to jumps have the same stationary distribution as the continuous-time process $\left\{X_{t}, t \geq 0\right\}$, which is $\operatorname{Erlang}(2, \lambda)$ by Theorem 3 . Therefore, the stationary distribution of $X(n)$ is equal to that of $V U$, where $V$ is $\operatorname{Erlang}(2, \lambda)$ distributed, $U$ is uniformly distributed on $(0,1)$, and $U$ and $V$ are independent. The stationary density of $X(n)$ is thus given by

$$
p(x)=\int_{x}^{\infty} \lambda^{2} v \mathrm{e}^{-\lambda v} \frac{1}{v} \mathrm{~d} v=\lambda \mathrm{e}^{-\lambda x}, \quad x>0 .
$$

We now compute the expected value and variance of $X(n)$. Notice that, for every $n \geq 1$,

$$
X(n)=U_{n} X(n-1)+\left(\tau_{n}-\tau_{n-1}\right) U_{n},
$$

where $X(0) \equiv X_{0}=0$. Furthermore, $\tau_{n}-\tau_{n-1} \stackrel{\mathrm{D}}{=} \operatorname{Exp}(\lambda), n \geq 1$. Hence, we can write

$$
X(n)=\sum_{i=1}^{n} R_{i} \tilde{W}_{i}^{(n)}, \quad n \geq 1,
$$

where $R_{1}, R_{2}, \ldots$ are i.i.d. and $\operatorname{Exp}(\lambda)$-distributed, $\tilde{W}_{i}^{(n)}=\prod_{j=i}^{n} U_{j}, i=1, \ldots, n$, and $R_{1}, R_{2}, \ldots$ are independent of $\left\{\tilde{W}_{i}^{(n)}, i=1, \ldots, n, n \geq 1\right\}$.

Theorem 7. For each $n \geq 1$,

$$
\mathrm{E}(X(n))=\frac{1}{\lambda}\left(1-\left(\frac{1}{2}\right)^{n}\right)
$$

and

$$
\operatorname{var}\{X(n)\}=\frac{1}{\lambda^{2}}\left(1-2\left(\frac{1}{2}\right)^{n}+2\left(\frac{1}{3}\right)^{n}-\left(\frac{1}{4}\right)^{n}\right)
$$


Proof. Since $R_{i}$ is independent of $W_{i}^{(n)}$, we have

$$
\begin{aligned}
\mathrm{E}(X(n)) & =\mathrm{E}\left(\sum_{i=1}^{n} R_{i} \tilde{W}_{i}^{(n)}\right) \\
& =\sum_{i=1}^{n} \mathrm{E}\left(R_{i}\right) \mathrm{E}\left(\tilde{W}_{i}^{(n)}\right) \\
& =\frac{1}{\lambda} \sum_{i=1}^{n}\left(\frac{1}{2}\right)^{n+1-i} \\
& =\frac{1}{\lambda}\left(1-\left(\frac{1}{2}\right)^{n}\right), \quad n \geq 1,
\end{aligned}
$$

namely (52).

To prove $(53)$, we let $\tilde{\boldsymbol{W}}^{(n)}=\left(\tilde{W}_{1}^{(n)}, \ldots, \tilde{W}_{n}^{(n)}\right)$ and compute the variance in two parts, according to

$$
\operatorname{var}\left\{\sum_{i=1}^{n} R_{i} \tilde{W}_{i}^{(n)}\right\}=\mathrm{E}\left(\operatorname{var}\left\{\sum_{i=1}^{n} R_{i} \tilde{W}_{i}^{(n)} \mid \tilde{\boldsymbol{W}}^{(n)}\right\}\right)+\operatorname{var}\left\{\mathrm{E}\left(\sum_{i=1}^{n} R_{i} \tilde{W}_{i}^{(n)} \mid \tilde{\boldsymbol{W}}^{(n)}\right)\right\} .
$$

Due to independence,

$$
\operatorname{var}\left\{\sum_{i=1}^{n} R_{i} \tilde{W}_{i}^{(n)} \mid \tilde{\boldsymbol{W}}^{(n)}\right\}=\frac{1}{\lambda^{2}} \sum_{i=1}^{n}\left(\prod_{j=i}^{n} U_{j}^{2}\right) .
$$

Hence,

$$
\mathrm{E}\left(\operatorname{var}\left\{\sum_{i=1}^{n} R_{i} \tilde{W}_{i}^{(n)} \mid \tilde{\boldsymbol{W}}^{(n)}\right\}\right)=\frac{1}{\lambda^{2}} \sum_{i=1}^{n}\left(\frac{1}{3}\right)^{n+1-i}=\frac{1}{2 \lambda^{2}}\left(1-\left(\frac{1}{3}\right)^{n}\right) .
$$

To calculate the second term of (54), we start with

$$
\mathrm{E}\left(\sum_{i=1}^{n} R_{i} \tilde{W}_{i}^{(n)} \mid \tilde{\boldsymbol{W}}^{(n)}\right)=\frac{1}{\lambda} \sum_{i=1}^{n} \tilde{W}_{i}^{(n)}
$$

It follows that

$$
\begin{aligned}
\operatorname{var}\left\{\mathrm{E}\left(\sum_{i=1}^{n} R_{i} \tilde{W}_{i}^{(n)} \mid \tilde{\boldsymbol{W}}^{(n)}\right)\right\} & =\frac{1}{\lambda^{2}} \operatorname{var}\left\{\sum_{i=1}^{n} \tilde{W}_{i}^{(n)}\right\} \\
& =\frac{1}{\lambda^{2}}\left[\sum_{i=1}^{n} \operatorname{var}\left\{\tilde{W}_{i}^{(n)}\right\}+2 \sum_{1 \leq i<j \leq n} \sum_{i \leq 0} \operatorname{cov}\left(\tilde{W}_{i}^{(n)}, \tilde{W}_{j}^{(n)}\right)\right] .
\end{aligned}
$$

In addition,

$$
\operatorname{var}\left\{\tilde{W}_{i}^{(n)}\right\}=\left(\frac{1}{3}\right)^{n+1-i}-\left(\frac{1}{4}\right)^{n+1-i}, \quad i=1, \ldots, n
$$


Similarly, for $i<j$,

$$
\operatorname{cov}\left(\tilde{W}_{i}^{(n)}, \tilde{W}_{j}^{(n)}\right)=\left(\frac{1}{2}\right)^{j-i}\left[\left(\frac{1}{3}\right)^{n+1-j}-\left(\frac{1}{4}\right)^{n+1-j}\right] .
$$

Substituting this into (56) and summing shows that

$$
\frac{1}{\lambda^{2}} \operatorname{var}\left\{\sum_{i=1}^{n} \tilde{W}_{i}^{(n)}\right\}=\frac{1}{\lambda^{2}}\left[\frac{1}{2}-2\left(\frac{1}{2}\right)^{n}+\frac{5}{2}\left(\frac{1}{3}\right)^{n}-\left(\frac{1}{4}\right)^{n}\right] .
$$

Finally, from (55), (56), and (57) we obtain (53).

Corollary 2. The asymptotic mean and variance of $X(n)$ are respectively

$$
\lim _{n \rightarrow \infty} \mathrm{E}(X(n))=\frac{1}{\lambda} \text { and } \lim _{n \rightarrow \infty} \operatorname{var}\{X(n)\}=\frac{1}{\lambda^{2}} .
$$

Finally, we develop recursive formulae for the distribution of $X(n), n=1,2, \ldots$ We start with the transition function

$$
K(y ; x)=\mathrm{P}(X(n) \leq y \mid X(n-1)=x)
$$

and its density $k(y ; x)$.

Theorem 8. For each $n \geq 1$,

$$
K(y ; x)=1-\exp \left(-\lambda(y-x)^{+}\right)+\lambda \mathrm{e}^{\lambda x} y \int_{\max [x, y]}^{\infty} \frac{1}{u} \mathrm{e}^{-\lambda u} \mathrm{~d} u,
$$

where $x^{+}=\max [x, 0]$, and

$$
k(y ; x)=\lambda \mathrm{e}^{\lambda x} \int_{\max [x, y]}^{\infty} \frac{1}{u} \mathrm{e}^{-\lambda u} \mathrm{~d} u .
$$

Proof. We have

$$
K(y ; x)=\mathrm{P}(X(n) \leq y \mid X(n-1)=x)=\mathrm{P}(U(x+R) \leq y),
$$

where $U \stackrel{\mathrm{D}}{=}$ uniform $(0,1), R \stackrel{\mathrm{D}}{=} \operatorname{Exp}(\lambda)$, and $U$ is independent of $R$. Notice that

$$
\mathrm{P}\left(U \leq \frac{y}{x+R} \mid R\right)=1(x+R \leq y)+1(x+R>y) \frac{y}{x+R} .
$$

This implies (58). Formula (59) is obtained by differentiating (58) with respect to $y$.

Let $f_{n}(x)$ denote the density function of $X(n)$. We can immediately prove that

$$
f_{1}(y)=\lambda \int_{y}^{\infty} \frac{1}{u} \mathrm{e}^{-\lambda u} \mathrm{~d} u, \quad 0<y<\infty .
$$

Theorem 9. For each $n \geq 2$,

$$
f_{n}(y)=f_{1}(y) \int_{0}^{y} \mathrm{e}^{\lambda x} f_{n-1}(x) \mathrm{d} x+\int_{y}^{\infty} \mathrm{e}^{\lambda x} f_{1}(x) f_{n-1}(x) \mathrm{d} x .
$$

Proof. For each $n \geq 2$,

$$
f_{n}(y)=\int_{0}^{\infty} k(y ; x) f_{n-1}(x) \mathrm{d} x .
$$

By substituting (59) into (61) we obtain (60). 


\section{The generalized uniform cut-off process and its relation to the shot noise process}

In this section we consider the following GCP, $\left(X_{t}\right)_{t \geq 0}$. As before, $X_{t}$ increases linearly, at rate 1 , between downward jumps. This time, however, the intervals $B_{1}, B_{2}, \ldots$ between the downward jumps are i.i.d. random variables with a general distribution with Laplace-Stieltjes transform $\beta(\cdot)$. The downward jump $Z_{n}$ after $B_{n}$ depends on $S_{n}$, where $S_{n}$ is the level of the $X_{t}$-process just before the $n$th jump. We generalize the uniform cut-off procedure of the previous sections in the following way. The remainder after the jump, $W_{n}=S_{n}-Z_{n}$, is given by $W_{n}=U^{a}\left(0, S_{n}\right)$, where $U^{a}(0, b)$ denotes a random variable with density $a t^{a-1} / b^{a}$ on $(0, b)$. (Of course, $a=1$ yields the uniform cut-off procedure.) We want to analyze $W_{n}$, the state of $X_{t}$ immediately after the $n$th jump. Since $S_{n}=W_{n}+B_{n}$, we have

$$
W_{n+1}=U^{a}\left(0, W_{n}+B_{n}\right), \quad n=1,2, \ldots
$$

We can show that the steady-state distribution of the $W_{n}$-process exists for all traffic parameters; see Remark 1, below.

It follows from (62) that the steady-state variable $W_{\mathrm{e}}$ of the sequence $W_{n}$ satisfies

$$
\begin{aligned}
\mathrm{E}\left(\mathrm{e}^{-\alpha W_{\mathrm{e}}}\right) & =\int_{0}^{\infty} \mathrm{e}^{-\alpha x} \int_{x}^{\infty} \frac{a x^{a-1}}{y^{a}} \mathrm{P}(W+B \in \mathrm{d} y) \mathrm{d} x \\
& =\int_{0}^{\infty} \frac{1}{y^{a}} \mathrm{P}(W+B \in \mathrm{d} y) \int_{0}^{y} a x^{a-1} \mathrm{e}^{-\alpha x} \mathrm{~d} x .
\end{aligned}
$$

We differentiate both sides of (63) with respect to $\alpha$ and use partial integration in the last integral, to obtain

$$
\frac{\mathrm{d}}{\mathrm{d} \alpha} \mathrm{E}\left(\mathrm{e}^{-\alpha W_{\mathrm{e}}}\right)=-a \frac{1-\beta(\alpha)}{\alpha} \mathrm{E}\left(\mathrm{e}^{-\alpha W_{\mathrm{e}}}\right) .
$$

The solution to this differential equation is readily verified to be given by

$$
\mathrm{E}\left(\mathrm{e}^{-\alpha W_{\mathrm{e}}}\right)=\exp \left\{-a \int_{0}^{\alpha} \frac{1-\beta(u)}{u} \mathrm{~d} u\right\} .
$$

Let us now point out a relation between the above growth-collapse process and the so-called shot noise process, which has been extensively studied in the literature on queueing models with workload-dependent service speed (see, e.g. [8], [13, pp. 558-559], and [17]). First transform $X_{t}$ into a 'dual' workload process following a procedure in [20]. This is done in two steps.

1. Construct a 'mountain' process by changing the negative jumps into negative slopes with gradient -1 .

2. Construct a workload process by changing the positive slopes into positive jumps of sizes $B_{1}, B_{2}, \ldots$

The resulting process has paths that are linearly decreasing between positive jumps; its workload just before the $n$th jump (which is of size $B_{n}$ ) is $W_{n}$.

Next consider the following shot noise process. Upward jumps, of sizes $B_{1}, B_{2}, \ldots$, occur after independent, $\operatorname{Exp}(\lambda)$-distributed time intervals. Between jumps, the process decreases at rate $r x$, where $x$ is the process level and $r>0$ is some constant. We can now interpret

$$
R(y, x)=\int_{y}^{x} \frac{1}{r w} \mathrm{~d} w
$$


as the time it takes for this shot noise process to decrease from level $x$ to level $y$, when no jumps occur.

Finally consider the following transformation:

$$
Y_{n}=-\frac{1}{r} \log \left(\frac{W_{n}}{S_{n}}\right) .
$$

A simple calculation shows that $Y_{n}$ is $\operatorname{Exp}(r a)$-distributed. Taking $a=\lambda / r$ results in $Y_{n}$ being $\operatorname{Exp}(\lambda)$-distributed. However, from (65) and (66) it is also clear that $Y_{n}$, as defined above, represents the time taken to decrease from $S_{n}$ to $W_{n}$ in the dual workload process, as well as an interarrival time in the shot noise process. It is thus seen that the level of the shot noise process just before the $n$th upward jump has exactly the same distribution as $W_{n}$ in the dual process and the original GCP.

The shot noise process just described has been analyzed in [8]. On p. 546 of [8] it was shown that it has a steady-state density $v(\cdot)$ whose LT is given by (64). Because of the PASTA property, this is also the LT of the density of the shot noise process just before jump epochs, and the above construction confirms that this is also the LT of $W_{\mathrm{e}}$. See pp. 546-547 of [8] for special cases (in which, for instance, $\lambda=r$ and $B_{1}$ is exponentially distributed, resulting in an exponential workload density).

Remark 1. The shot noise process not only never reaches 0 , but is also known to be stable for all offered traffic loads. The relation to the special process described above implies that the same properties hold for this latter process.

\section{The Markov-modulated case}

Now let us look at the Markov-modulated case, as described in Section 1. The underlying Markov process is two-dimensional: $Z_{t}=\left(X_{t}, J_{t}\right)$; and the generator is

$$
\begin{aligned}
(g f)(x, i)= & c(i) f^{\prime}(x, i)+\sum_{j \neq i} q_{i j} f(x, j)-\left(q_{i}+r(x)\right) f(x, i) \\
& +r(x) \int_{[0, x)} f(y, i) \mu_{x}(\mathrm{~d} y), \quad i=1, \ldots, n,
\end{aligned}
$$

or, in matrix form,

$$
(g \boldsymbol{f})(x)=\boldsymbol{C} \boldsymbol{f}^{\prime}(x)+(\boldsymbol{Q}-r(x) \boldsymbol{E}) \boldsymbol{f}(x)+r(x) \boldsymbol{D}_{f}(x),
$$

where

(a) $\boldsymbol{f}(x)=(f(x, 1), \ldots, f(x, n))^{\top}$;

(b) $\boldsymbol{Q}=\left(q_{i j}\right)_{i, j \in\{1, \ldots, n\}}$ is the generator of the Markov chain $J_{t}$, and $q_{i}=-q_{i i}$;

(c) $\boldsymbol{C}$ and $\boldsymbol{D}_{f}(x)$ are diagonal matrices with diagonal entries $c(i)$ and $\int_{[0, x)} f(y, i) \mu_{x}(\mathrm{~d} y)$, respectively;

(d) $\boldsymbol{E}$ is the $n \times n$ identity matrix.

We can now derive the stationary distribution of $Z_{t}$ and the LT $\mathrm{E}_{x}\left(\mathrm{e}^{-\alpha T_{a}}\right)$ in terms of integral and differential equations. 
Instead of developing this generalization, we finally consider the following problem: what is the distribution of $T_{a} \wedge \sigma$ ? To see that this is a relevant question, let us, for example, interpret $\sigma$ as the time at which disaster occurs in some 'system', say some technical item. Assume that the system has to run indefinitely; upon any disaster it has to be replaced by a new, identical one, but it can also be replaced preventively when its age reaches a certain threshold $a>0$, which has to be specified by the controller. Thus, the first replacement takes place at time $T_{a} \wedge \sigma$. Suppose that after replacement the modulating chain is restarted at some fixed state $i_{0}$. If a replacement of a functional system costs $\$ C_{1}$ and a replacement upon disaster (of a thus nonfunctional system) costs $\$ C_{2}$ (where $C_{1}<C_{2}$ ), then the long-run average cost of running the system when using the policy $T_{a}$ is given by

$$
C(a)=\frac{C_{1} \mathrm{P}_{0, i_{0}}\left(T_{a} \wedge \sigma=T_{a}\right)+C_{2}\left(1-\mathrm{P}_{0, i_{0}}\left(T_{a} \wedge \sigma=T_{a}\right)\right)}{\mathrm{E}_{0, i_{0}}\left(T_{a} \wedge \sigma\right)},
$$

where $\mathrm{P}_{x, i}(\cdot)$ and $\mathrm{E}_{x, i}(\cdot)$ respectively denote conditional probability and conditional expectation given that $Z_{0}=(x, i)$. Hence, $\mathrm{P}_{x, i}\left(T_{a} \wedge \sigma=T_{a}\right)$ and $\mathrm{E}_{x, i}\left(T_{a} \wedge \sigma\right)$ are important quantities; once they are known as functions of $a$ we can try to minimize $C(a)$.

We deal with this problem as follows. Suppose that the process is killed at time $\sigma$ by entering a coffin state $\partial$. By Dynkin's formula, we have

$$
f(x, i)=\mathrm{E}_{x, i}\left(\int_{0}^{T}(g f)\left(Z_{t}\right) \mathrm{d} t\right)-\mathrm{E}_{x, i}\left(f\left(Z_{T}\right)\right)
$$

for a bounded function $f$ in the domain of $g$ and any integrable stopping time $T$. Now apply (67) to $T=T_{a} \wedge \sigma$ in two cases:

(i) $f=f_{1}$, such that $\left(g f_{1}\right)(x, i)=0, x \in(0, a)$, with $f_{1}(\partial, i)=0$ and $f_{1}(a, i)=1$.

(ii) $f=f_{2}$, such that $\left(g f_{2}\right)(x, i)=-1, x \in(0, a)$, with $f_{2}(\partial, i)=0$ and $f_{2}(a, i)=0$.

A moment's reflection shows that if $f_{1}$ and $f_{2}$ have these properties, then

$$
\begin{aligned}
& f_{1}(x, i)=\mathrm{P}_{x, i}\left(T_{a} \wedge \sigma=T_{a}\right), \\
& f_{2}(x, i)=\mathrm{E}_{x, i}\left(T_{a} \wedge \sigma\right) .
\end{aligned}
$$

Hence, we have to solve

$$
\boldsymbol{C} \boldsymbol{f}^{\prime}(x)+(\boldsymbol{Q}-r(x) \boldsymbol{E}) \boldsymbol{f}(x)=(0, \ldots, 0)^{\top},
$$

subject to the condition that

$$
\boldsymbol{f}(a, \cdot)=(1, \ldots, 1)^{\top}
$$

and

$$
\boldsymbol{C} \boldsymbol{f}^{\prime}(x)+(\boldsymbol{Q}-r(x) \boldsymbol{E}) \boldsymbol{f}(x)=(-1, \ldots,-1)^{\top},
$$

subject to the condition that

$$
\boldsymbol{f}(a, \cdot)=(0, \ldots, 0)^{\top} .
$$

Let us finally show that, for $n=2$ (when there are two modulating states), these systems of linear differential equations can be solved in special cases. 
Example 5. Let $n=2$ and set $q_{12}=\mu_{1}, q_{21}=\mu_{2}$, and $f(x, i)=h_{i}(x), i=1,2$. Then, for $x \in(0, a],(68)$ and (69) become

$$
\begin{aligned}
& c(1) h_{1}^{\prime}(x)-\left(\mu_{1}+\lambda(x)\right) h_{1}(x)+\mu_{1} h_{2}(x)=0, \\
& c(2) h_{2}^{\prime}(x)-\left(\mu_{2}+\lambda(x)\right) h_{2}(x)+\mu_{2} h_{1}(x)=0,
\end{aligned}
$$

subject to

$$
h_{1}(a)=h_{2}(a)=1
$$

and (70) and (71) become

$$
\begin{aligned}
& c(1) h_{1}^{\prime}(x)-\left(\mu_{1}+\lambda(x)\right) h_{1}(x)+\mu_{1} h_{2}(x)=-1, \\
& c(2) h_{2}^{\prime}(x)-\left(\mu_{2}+\lambda(x)\right) h_{2}(x)+\mu_{2} h_{1}(x)=-1,
\end{aligned}
$$

subject to

$$
h_{1}(a)=h_{2}(a)=0 .
$$

Formulae (72)-(73) can be transformed into the two second-order linear differential equations

$$
\begin{aligned}
0= & c(1) c(2) h_{i}^{\prime \prime}(x)-\left[(c(1)+c(2)) \lambda(x)+\mu_{1} c(2)+\mu_{2} c(1)\right] h_{i}^{\prime}(x) \\
& +\left[\lambda(x)^{2}+\left(\mu_{1}+\mu_{2}\right) \lambda(x)\right] h_{i}(x), \quad i=1,2, x \in(0, a],
\end{aligned}
$$

with the boundary conditions

$$
h_{i}(a)=1, \quad h_{i}^{\prime}(a)=\lambda(a), \quad i=1,2 .
$$

Similarly, (74)-(75) lead to

$$
\begin{aligned}
0= & c(1) c(2) h_{i}^{\prime \prime}(x)-\left[(c(1)+c(2)) \lambda(x)+\mu_{1} c(2)+\mu_{2} c(1)\right] h_{i}^{\prime}(x) \\
& +\left[\lambda(x)^{2}+\left(\mu_{1}+\mu_{2}\right) \lambda(x)\right] h_{i}(x)-\mu_{1}-\mu_{2}-\lambda(x), \quad i=1,2, x \in(0, a],
\end{aligned}
$$

with the boundary conditions

$$
h_{i}(a)=0, \quad h_{i}^{\prime}(a)=-1, \quad i=1,2 .
$$

Now consider the proportional jump intensity $r(x)=\lambda x$, which we assumed to hold in Section 3. In this case, (76) takes the form

$$
h_{i}^{\prime \prime}(x)+\left[d_{1} x+d_{2}\right] h_{i}^{\prime}(x)+\left[d_{3} x^{2}+d_{4} x\right] h_{i}(x)=0, \quad i=1,2, x \in(0, a],
$$

where

$$
\begin{aligned}
& d_{1}=-(c(1) c(2))^{-1}[c(1)+c(2) \lambda x], \\
& d_{2}=-(c(1) c(2))^{-1}\left[\mu_{1} c(2)+\mu_{2} c(1)\right], \\
& d_{3}=(c(1) c(2))^{-1} \lambda^{2} \\
& d_{4}=(c(1) c(2))^{-1}\left(\mu_{1}+\mu_{2}\right) \lambda .
\end{aligned}
$$

The general solution to (80) can be given as a linear combination of the degenerate hypergeometric functions $\Phi$ and $\Psi$ that we used in Section 3 (see [21, Equation 2.1.2.103]), for certain 
arguments, and the boundary conditions (77) then uniquely determine the coefficients. We will not write down the exact formulae, as they are lengthy. Let $\Phi_{0}(x)$ and $\Psi_{0}(x)$ be two linearly independent solutions to (80). The corresponding general solution to the inhomogeneous equation (78) is given by

$$
\begin{aligned}
C_{1} \Phi_{0}(x)+C_{2} \Psi_{0}(x)+(c(1) c(2))^{-1} & {\left[\Psi_{0}(x) \int_{0}^{x} \frac{\Phi_{0}(u)\left(\mu_{1}+\mu_{2}+\lambda(u)\right)}{W(u)} \mathrm{d} u\right.} \\
& \left.-\Phi_{0}(x) \int_{0}^{x} \frac{\Psi_{0}(u)\left(\mu_{1}+\mu_{2}+\lambda(u)\right)}{W(u)} \mathrm{d} u\right],
\end{aligned}
$$

where $W(u)=\Phi_{0}(u) \Psi_{0}^{\prime}(u)-\Psi_{0}(u) \Phi_{0}^{\prime}(u)$ and the constants $C_{1}$ and $C_{2}$ can be determined from (79).

\section{References}

[1] Albrecher, H. and Boxma, O. J. (2004). A ruin model with dependence between claim sizes and claim intervals. Insurance Math. Econom. 35, 245-254.

[2] Albrecher, H. And Boxma, O. J. (2005). On the discounted penalty function in a Markov-dependent risk model. Insurance Math. Econom. 37, 650-672.

[3] Altman, E., Avrachenkov, K., Barakat, C. and Núñez-Queija, R. (2001). State-dependent $M / G / 1$ type queueing analysis for congestion control in data networks. In Proc. IEEE INFOCOM 2001 (Anchorage, AK, April 2001), IEEE, Piscataway, NJ, pp. 1350-1359.

[4] Altman, E., Avrachenkov, K., Kherani, A. A. and Prabhu, B. J. (2005). Performance analysis and stochastic stability of congestion control protocols. In Proc. IEEE INFOCOM 2005 (Miami, FL, March 2005), eds K. Makki and E. Knightly, IEEE, Piscataway, NJ.

[5] Asmussen, S. (2000). Ruin Probabilities (Adv. Ser. Statist. Sci. Appl. Prob. 2). World Scientific, Singapore.

[6] Asmussen, S. (2003). Applied Probability and Queues, 2nd edn. Springer, New York.

[7] BAK, P. (1996). How Nature Works: The Science of Self-Organized Criticality. Copernicus, New York.

[8] Bekker, R., Borst, S. C., Boxma, O. J. and Kella, O. (2003). Queues with workload-dependent arrival and service rates. Queueing Systems 46, 537-556.

[9] Boxma, O. J. AND Perry, D. (2001). A queueing model with dependence between service and interarrival times. Europ. J. Operat. Res. 128, 611-624.

[10] Boxma, O. J., Kella, O. and Perry, D. (2001). An intermittent fluid system with exponential on-times and semi-Markov input rates. Prob. Eng. Inf. Sci. 15, 189-198.

[11] Boxma, O. J., Kaspi, H., Kella, O. and Perry, D. (2005). On/off storage systems with state-dependent input, output and switching rates. Prob. Eng. Inf. Sci. 19, 1-14.

[12] Budhiraja, A., Hernandez-Campos, F., Kulkarni, V. G. and Smith, F. D. (2004). Stochastic differential equation for TCP window size: analysis and experimental validation. Prob. Eng. Inf. Sci. 18, 111-140.

[13] Cohen, J. W. (1982). The Single Server Queue. North-Holland, Amsterdam.

[14] Davis, M. H. A. (1984). Piecewise-deterministic Markov processes: a general class of non-diffusion stochastic models. J. R. Statist. Soc. B 46, 353-388.

[15] Davis, M. H. A. (1993). Markov Models and Optimization. Chapman and Hall, London.

[16] Eliazar, I. And Klafter, J. (2004). A growth-collapse model: Lévy inflow, geometric crashes, and generalized Ornstein-Uhlenbeck dynamics. Physica A 334, 1-21.

[17] Keilson, J. and Mermin, N. D. (1959). The second-order distribution of integrated shot noise. IRE Trans. Inf. Theory 5, 75-77.

[18] Kella, O. and Stadje, W. (2001). On hitting times for compound Poisson dams with exponential jumps and linear release. J. Appl. Prob. 38, 781-786.

[19] Kherani, A. A. AND Kumar, A. (2002). Stochastic models for throughput analysis of randomly arriving elastic flows in the Internet. In Proc. IEEE INFOCOM 2002 (New York, June 2002), IEEE, Piscataway, NJ.

[20] Perry, D. and Stadje, W. (2003). Duality of dams via mountain processes. Operat. Res. Lett. 31, 451-458.

[21] Polyanin, A. D. and Zaitsev, V. F. (1995). Handbook of Exact Solutions for Ordinary Differential Equations. CRC, Boca Raton, FL.

[22] Rolski, T., Schmidli, H., Schmidt, V. And Teugels, J. (1999). Stochastic Processes for Insurance and Finance. John Wiley, New York.

[23] Scheinhardt, W., van Foreest, N. and Mandjes, M. (2005). Continuous feedback fluid queues. Operat. Res. Lett. 33, 551-559. 